\title{
Are "Patellofemoral Symptoms" Truly Related to the Patellofemoral Joint?
}

\author{
Rafik Yassa, MRCS ${ }^{1}$, Mahdi Yacine Khalfaoui, $\mathrm{MRCS}^{2}$, and Andrew P. Davies, FRCS (T\&O) ${ }^{3}$ \\ ${ }^{1}$ Department of Trauma and Orthopaedics, North Manchester General Hospital, ${ }^{2}$ Central Manchester Teaching Hospitals, Manchester, \\ ${ }^{3}$ Department of Trauma and Orthopaedics, Morriston Hospital, Swansea, UK
}

\begin{abstract}
Purpose: The pattern of symptoms of knee osteoarthritis has been thought to be indicative of specific compartment involvement. This study investigated whether there was a true correlation between patellofemoral joint (PFJ) symptoms and unicompartmental patellofemoral arthritis.

Materials and Methods: A prospective analysis of 34 patients rendered to be suffering from PFJ osteoarthritis and subsequently undergoing unicompartmental patellofemoral arthroplasty (PFA) was performed. A control cohort of 32 patients suffering from medial tibiofemoral joint (MTFJ) osteoarthritis was included in the analysis. Four questions derived from the Oxford knee score questionnaire, traditionally deemed to be indicative of PFJ osteoarthritis, were combined to create a PFJ subscore and statistically analyzed for their potential relationship with PFJ osteoarthritis and outcomes following PFA.

Results: The PFJ subscore indicated slightly worse pathology in patients undergoing PFA compared to MTFJ replacement, but the difference was not significant (9.7 and 9.6, respectively; $\mathrm{p}=0.851$ ). The improvement in PFJ symptoms was higher in those undergoing PFA compared to MTFJ surgery; however, the difference was not statistically significant (3.7 and 2.2 respectively, $\mathrm{p}=0.074$ ).

Conclusions: We were unable to prove that these symptoms were predictive of PFJ pathology in the preoperative setting, nor were they useful in interpreting which symptoms would likely improve following PFA.
\end{abstract}

Keywords: Knee, Osteoarthritis, Patellofemoral, Unicompartmental arthroplasty

\section{Introduction}

Osteoarthritis of the knee is a common and disabling condition. All or any of the three compartments may be affected in any combination; however, isolated unicompartmental disease of the medial tibiofemoral joint (MTFJ) and patellofemoral joint (PFJ) are common patterns ${ }^{1)}$. The debate as to whether to offer total knee replacement or unicompartmental replacement in such cases remains controversial.

Received May 19, 2015; Revised (1st) September 25, 2015;

(2nd) October 26, 2015; (3rd) October 27, 2015;

Accepted December 4, 2015

Correspondence to: Rafik Yassa, MRCS

Department of Trauma and Orthopaedics, North Manchester General

Hospital, Delaunays Roadm, Crumpsall, M8 5RB, UK.

Tel: +44-0161-720-2302, Fax: +44-0161-720-2399

E-mail: rafikragai@yahoo.com

This is an Open Access article distributed under the terms of the Creative Commons Attribution Non-Commercial License (http://creativecommons.org/licenses/by-nc/4.0/) which permits unrestricted non-commercial use, distribution, and reproduction in any medium, provided the original work is properly cited.
Treatment for single compartment pathology ranges widely across the literature. Techniques described include arthroscopic debridement $^{2)}$, high tibial osteotomy ${ }^{3,4)}$, and unicompartmental knee replacement both for the PFJ and MTFJ ${ }^{5)}$. All have been described to have variable outcomes with only total knee arthroplasty (TKA) consistently demonstrating excellent long-term results $^{6}$. Some evidence has even suggested that unicompartmental arthroplasty offers superior results in appropriately selected cases $^{7}$.

It is widely agreed that patient selection is a crucial step in the decision-making when considering unicompartmental knee arthroplasty (UKA). The pattern of symptoms has often been advocated as predicting which compartment is involved in the disease process. Biomechanically it is believed that the PF compartment is subjected to increased load during activities involving knee flexion and quadriceps muscle contraction such as ascending or descending stairs and rising from sitting position or sitting ${ }^{8)}$. Based on this concept, it is widely believed that patients with isolated PFJ disease are most likely to complain of pain when performing these activities. There were no available studies in the 
current literature confirming this common belief. Furthermore, we did not identify research correlating the presenting symptoms with the intraoperative findings or patient outcomes following unicompartmental arthroplasty of the PFJ.

Our study was, therefore, designed to investigate whether patients with unicompartmental osteoarthritis of the PFJ could truly be identified during their initial presentation solely based on the detailed symptomatology identified during the history-taking phase of assessment. Furthermore, we wanted to assess whether PFJ symptoms specifically improved following treatment with unicompartmental arthroplasty targeted at the PFJ only as our secondary outcome in this study.

\section{Materials and Methods}

It is the senior author's (Davies) practice to offer unicompartmental arthroplasty in appropriate cases. The final decision to proceed to unicompartmental or total arthroplasty is made after a medial parapatellar arthrotomy followed by a full inspection of the articular surfaces of the three compartments. In the event of isolated involvement of a single compartment confirmed during the procedure, either a patellofemoral or tibiofemoral joint arthroplasty is performed. All patients filled in the Oxford knee score (OKS) questionnaire independently preoperatively and again at routine follow-up appointments on average at 1 year postoperatively. Local Ethical Committee approval was sought and obtained before the start of the study.

Patients were recruited into the study consecutively if they met our inclusion criteria. We included all patients who were diagnosed with single compartment osteoarthritis (PFJ and MTFJ) following a focused history taking, clinical/radiological examination. All of the included patients consented for partial and total knee replacement prior to the surgical procedure. Data were collected prospectively for a total of 66 UKA cases for osteoarthritis during the 5-year study period. A total of 34 patients rendered to be suffering from unicompartmental PFJ osteoarthritis underwent patellofemoral arthroplasty (PFA). During the same study period, a control group of 32 cases were deemed to have isolated MTFJ involvement and, therefore, subsequently underwent medial tibiofemoral arthroplasty (MTFA). All patients were examined preoperatively and operated on directly by or under the direct supervision of the senior author in a single centre. Clinical examination particularly focused on the integrity of the surrounding knee ligaments, with any ligament deficiency excluding patients from our study. Furthermore, if it was thought that arthropathy of the PFJ was related to patellar instability, patients would be initially investigated for anatomical muscular, ligamentous and osseous factors and treated accordingly in order to correct for instability first and, therefore, excluded from our study.

We used the Femoro-Patella Vialla cemented prosthesis (Wright Medical Technology Inc., Arlington, TN, USA) for the PFA cohort and the Miller-Galante fixed-bearing cemented prosthesis (Zimmer, Warsaw, IN, USA) for the MTFA group. All operations were performed using a standard midline incision and medial parapatellar approach. The articular surfaces of all three compartments and the anterior cruciate ligament (ACL) were inspected during the surgery. Furthermore, during the procedures investigated in this study, particular note was taken to assess for meniscal injury, as this would clearly act to confound the symptoms relating to PFJ pathology. Unicompartmental arthroplasty was performed for isolated unicompartmental disease only. Cases of isolated PF arthropathy with meniscal involvement requiring treatment would be excluded from later analysis. In cases with multiple compartment involvement, it was a standard practice to proceed onto TKA and these patients would also be excluded from later analysis. All operations were performed using a high thigh tourniquet and prophylactic antibiotics on induction of anaesthesia. All patients had calf pumps during the surgery and thromboembolic deterrent stockings together with $150 \mathrm{mg}$ of Aspirin daily for 6 weeks postoperatively. No drains were used for any of the patients involved in this study. Physiotherapy was commenced on the first postoperative day with all patients encouraged to fully weight bear. Unrestricted knee range of movement was permitted. Knee bandages were removed at 48 hours and skin clips were removed a total of 12 days following the procedure.

The $\mathrm{OKS}^{9)}$ is widely regarded as the gold standard patient-recorded outcome measure across the United Kingdom for all varieties of knee arthroplasty procedures. A selection of 4 questions, derived directly from the OKS, was used for their traditional association with isolated PFJ pathology (Table 1).

Each question had a response range from 0 to 4 . A score of 0 denoted the best outcome with relation to the question and 4 the worst outcome. The selected questions were used to develop a 16-point subscore from the OKS designed specifically to assess the PFJ osteoarthritis in patients initially presenting with knee pathology (PFJ subscore). The score was also calculated postoperatively in order to evaluate the degree of improvement in PFJ symptoms after targeted unicompartmental arthroplasty as a secondary indicator of whether the subscore was representative of PFJ osteoarthritis. The overall OKS was also calculated with 0 as the best score and 48 as the worst possible outcome. Relative 
comparisons were conducted between the PFJ subscore and the OKS. Each of the questions making up the PFJ subscore was analysed independently.

The MTFJ cohort was used as our control group for comparison in the event of positive statistically significant findings related to the PFJ subscore. A combination of the paired and unpaired $t$-test was utilised for matched pair and independent mutually distributed data, respectively. The widely accepted threshold of $<0.05$ was considered to be statistically significant. All data were analysed using Stata ver. 8.2 (Stata Corp., College Station, TX, USA) and Microsoft Excel (Microsoft, Redmond, WA, USA).

Table 1. Selection of Questions from the Oxford Knee Score Denoting Patellofemoral Joint Pathology

\begin{tabular}{cc}
\hline No. & Question \\
\hline 1 & After a meal (sat at the table) how painful has it been for \\
& you to stand up from the chair because of your knee? \\
2 & Could you kneel down and get up again afterwards? \\
3 & Have you felt that your knee might suddenly give way \\
& or let you down? \\
4 & Could you walk down a flight of stairs? \\
\hline
\end{tabular}

\section{Results}

A total of 66 cases of UKA were analysed across 58 patients (8 bilateral cases). Data was complete for all patients and there was no loss to follow-up. The average age of patients included in the study was 57 years (range, 52 to 63 years), with 51 males and 7 females. The average follow-up periods were 11.0 and 10.7 months for the PFJ and MTFJ groups, respectively.

The mean total preoperative OKS scores were 28.2 with an overall improvement of 10.0 and 28.6 with an improvement of 9.1, for PF and MTFA, respectively, which were considered to be statistically significant $(\mathrm{p}=0.000)$ (Table 2$)$. The overall PFJ subscore similarly improved significantly postoperatively in our PFJ and MTFJ cohorts. Individual question sub-analysis revealed similar statistically significant improvements across all 4 questions in the PFJ patients and in all but question 2 (kneeling) of the MTFJ cohort, where the postoperative improvement was not significant $(\mathrm{p}=0.773)$ (Table 2).

Analysis comparing the preoperative and overall improvement in OKS for PFJ pathology against our control group (MTFJ pathology) revealed no significant difference between the groups ( $\mathrm{p}=0.849$ and $\mathrm{p}=0.730$, respectively) (Table 3 ).

Table 2. Comparison of Mean Preoperative (Preop) and Postoperative (Postop) Scores

\begin{tabular}{|c|c|c|c|c|c|}
\hline Score (maximal score) & Preop & Postop & Difference & p-value ${ }^{a)}$ & $95 \% \mathrm{CI}$ \\
\hline \multicolumn{6}{|l|}{ Oxford knee score (48) } \\
\hline PFA & 28.2 & 18.2 & 10.0 & 0.000 & 6.89 to 9.97 \\
\hline MTFA & 28.6 & 19.5 & 9.1 & 0.000 & 4.91 to 13.28 \\
\hline \multicolumn{6}{|l|}{ PFJ subscore (16) } \\
\hline PFA & 9.7 & 6.0 & 3.7 & 0.000 & 2.75 to 4.66 \\
\hline MTFA & 9.6 & 7.3 & 2.2 & 0.003 & 0.83 to 3.61 \\
\hline \multicolumn{6}{|l|}{ Standing from chair (4) } \\
\hline PFA & 2.3 & 1.4 & 0.9 & 0.000 & 0.50 to 1.38 \\
\hline MTFA & 2.2 & 1.5 & 0.7 & 0.002 & 0.26 to 1.11 \\
\hline \multicolumn{6}{|l|}{ Kneeling (4) } \\
\hline PFA & 3.2 & 2.7 & 0.5 & 0.016 & 0.11 to 0.95 \\
\hline MTFA & 2.9 & 2.8 & 0.1 & 0.773 & -0.38 to 0.50 \\
\hline \multicolumn{6}{|l|}{ Giving way (4) } \\
\hline PFA & 2.0 & 0.8 & 1.2 & 0.000 & 0.76 to 1.65 \\
\hline MTFA & 2.2 & 1.5 & 0.7 & 0.015 & 0.15 to 1.29 \\
\hline \multicolumn{6}{|l|}{ Walking downstairs (4) } \\
\hline PFA & 2.2 & 1.2 & 1.0 & 0.000 & 0.69 to 1.37 \\
\hline MTFA & 2.3 & 1.5 & 0.6 & 0.001 & 0.33 to 1.17 \\
\hline
\end{tabular}

CI: confidence interval, PFA: patellofemoral arthroplasty, MTFA: medial tibiofemoral arthroplasty, PFJ: patellofemoral joint.

${ }^{\text {a) }}$ Paired $t$-test was used to compare dependant scores. 
Analysis looking at the PFJ subscore suggested that there was no significant difference between PFJ and MTFJ arthritis.

Postoperatively, the average improvement in the PFJ subscore was 3.7 in comparison to just 2.2 in the MTFJ group, suggesting the subscore was predictive of PFJ pathology; however, this was again not statistically significant $(\mathrm{p}=0.074)$.

Individual analysis of the preoperative scores for the four questions comprising the PFJ subscore revealed that there were no significant differences between our PFJ and MTFJ cohorts (Table 3). Furthermore, we were unable to demonstrate any significant differences in the postoperative improvements for each individual question between the PFJ and MTFJ groups (Table 3).

In the final part of our analysis, we compared the relative OKS score with the PJF subscore to identify whether the subscore was more related to PFJ patients than the traditionally utilised 48-point OKS. For our PFJ cohort, the relative preoperative PFJ subscore was slightly worse than the relative OKS, suggesting the subscore was related to PFJ pathology. This, however, failed to meet our significance threshold $(\mathrm{p}=0.188)$ (Table 4$)$. This analysis was repeated for relative improvements in the OKS and PFJ subscore. Again improvement in the subscore was insignificantly higher than the relative improvement in the OKS in patients with $\mathrm{PFJ}$ pathology $(\mathrm{p}=0.240)$, suggesting a further possible relationship of these symptoms to actual PFJ pathology. Interestingly, when similar analysis was performed on the MTFJ cohort of patients, the relative improvement in the OKS was significantly higher than the PFJ subscore $(\mathrm{p}=0.033)$, indicating an inverse relationship of the subscore with MTFJ patients.

Table 3. Comparison of Patellafemoral Arthroplasty (PFA) and Medial Tibiofemoral Arthroplasty (MTFA) Groups

\begin{tabular}{|c|c|c|c|c|}
\hline Score (maximal score) & PFA & MTFA & p-value ${ }^{a}$ & $95 \% \mathrm{CI}$ \\
\hline \multicolumn{5}{|l|}{ Oxford knee score (48) } \\
\hline Preop & $28.2 \pm 8.8$ (5 to 42$)$ & $28.6 \pm 8.9(4$ to 44$)$ & 0.849 & -4.77 to 3.94 \\
\hline Difference & $10.0 \pm 11.6(-4$ to 27$)$ & $9.1 \pm 11.6(-13$ to 31$)$ & 0.730 & -4.18 to 5.93 \\
\hline \multicolumn{5}{|l|}{ PFJ subscore (16) } \\
\hline Postop $^{\text {b) }}$ & $6.0 \pm 2.8$ ( 2 to 12$)$ & $7.3 \pm 3.2(1$ to 13$)$ & 0.070 & -2.80 to 0.11 \\
\hline Difference & $3.7 \pm 2.7(-2$ to 10$)$ & $2.2 \pm 3.9(-5$ to 13$)$ & 0.074 & -0.15 to 3.12 \\
\hline \multicolumn{5}{|l|}{ Standing from chair (4) } \\
\hline Preop & $2.3 \pm 1.1(0$ to 4$)$ & $2.2 \pm 1.0(0$ to 41$)$ & 0.775 & -0.45 to 0.60 \\
\hline Postop $^{\text {b) }}$ & $1.4 \pm 1.1(0$ to 4$)$ & $1.5 \pm 1.0(0$ to 3$)$ & 0.478 & -0.68 to 0.32 \\
\hline Preop & $3.2 \pm 0.9(0$ to 4$)$ & $2.9 \pm 0.9(1$ to 4$)$ & 0.227 & -0.17 to 0.71 \\
\hline Postop $^{\text {b) }}$ & $2.7 \pm 1.0(1$ to 4$)$ & $2.8 \pm 1.1(0$ to 4$)$ & 0.466 & -0.73 to 0.34 \\
\hline Difference & $0.5 \pm 1.2(-3$ to 3$)$ & $0.1 \pm 1.2(-2$ to 3$)$ & 0.123 & -0.13 to 1.06 \\
\hline \multicolumn{5}{|l|}{ Giving way (4) } \\
\hline Preop & $2.0 \pm 1.2(0$ to 4$)$ & $2.2 \pm 1.4(0$ to 4$)$ & 0.623 & -0.80 to 0.48 \\
\hline Postop $^{\text {b) }}$ & $0.8 \pm 0.8(0$ to 3$)$ & $1.5 \pm 1.1(0$ to 4$)$ & 0.008 & -1.12 to -0.17 \\
\hline Difference & $1.2 \pm 1.3(-1$ to 4$)$ & $0.7 \pm 1.6(-2$ to 4$)$ & 0.170 & -0.21 to 1.19 \\
\hline \multicolumn{5}{|l|}{ Walking downstairs (4) } \\
\hline Preop & $2.2 \pm 0.9(0$ to 4$)$ & $2.3 \pm 0.7(0$ to 3$)$ & 0.829 & -0.45 to 0.36 \\
\hline
\end{tabular}

Values are presented as mean \pm standard deviation (range).

CI: confidence interval, Preop: preoperative, Postop: postoperative.

${ }^{\text {a) }}$ Unpaired $t$-test was used to compare the two independent cohorts.

${ }^{b)}$ Average postoperative follow-up periods were 11.0 (PFA) and 10.7 (MTFA) months. 
Table 4. Comparison of Traditional Oxford Knee Score (OKS) and Patellofemoral Joint (PFJ) Subscore

\begin{tabular}{|c|c|c|c|c|c|c|c|c|}
\hline \multirow{2}{*}{ Score } & \multicolumn{4}{|c|}{ PFA } & \multicolumn{4}{|c|}{ MTFA } \\
\hline & OKS & PFJ subscore & p-value $e^{a)}$ & $95 \% \mathrm{CI}$ & OKS & PFJ subscore & p-value $e^{a)}$ & $95 \% \mathrm{CI}$ \\
\hline \multicolumn{9}{|c|}{ Preoperative scores } \\
\hline Relative values & 0.59 & 0.61 & 0.188 & -0.050 to 0.01 & 0.60 & 0.60 & 0.886 & -0.03 to 0.03 \\
\hline \multicolumn{9}{|c|}{ Improvement in outcomes } \\
\hline Relative values & 0.21 & 0.23 & 0.240 & -0.07 to 0.02 & 0.19 & 0.14 & 0.033 & 0.00 to 0.10 \\
\hline
\end{tabular}

PFA: patellofemoral arthroplasty, MTFA: medial tibiofemoral arthroplasty, CI: confidence interval.

${ }^{\text {a) }}$ Paired $t$-test was used to compare relative scores.

\section{Discussion}

Treatment of unicompartmental knee osteoarthritis remains controversial. Some surgeons offer their patients TKA regardless of the number of the compartments involved, while others consider unicompartmental arthroplasty in carefully selected patients. The decision making process varies from one surgeon to the other. Some surgeons base their decision on the radiographic appearance of osteoarthritis in combination with the clinical presentation. While others take the final decision intraoperatively after opening the knee joint and assessing the three compartments individually. Advocates of unicompartmental arthroplasty base their decision on higher percentage of excellent outcomes and the preservation of overall bone stock. Some studies have confirmed that if unicompartmental arthroplasty is suitable for the case, then it offers superior long-term outcomes to total arthroplasty $^{7)}$. Joint registries, however, indicate that the revision rate for unicompartmental arthroplasty is higher than that for total joint arthroplasty ${ }^{10)}$. There is evidence that TKA offers a more predicable outcome with fewer excellent results but also fewer clinical failures ${ }^{11)}$. Most recently, there has been an upsurge in UKA interest with the advent of minimally invasive techniques, resulting in minimal trauma, closer kinematics to the original knee, and quicker recovery ${ }^{12)}$.

Our clinical question is unique and has not been asked before according to our literature review. We felt an important determinant when selecting patients for PFA was through questioning patients on specific symptoms widely accepted as representative of PFJ pathology. For this reason, determining whether these questions were truly representative of this pathology was a relevant study subject. Furthermore, early clinical failure of unicompartmental arthroplasty and resultant persistent pain are usually attributed to the un-replaced compartments. Therefore, accurately isolating compartment pathology can be useful not only in the preoperative setting but also postoperatively when a single compartment only has been replaced.

The kinematics across the patellofemoral and tibiofemoral joints differs considerably and results in significant differences in the overall joint reaction forces. The joint reaction force across the PFJ is highest with the knee partially flexed. For this reason, activities involving standing from a sitting position, kneeling, and walking downstairs are all thought to exacerbate PFJ symptoms. Furthermore, "giving way" of the knee has been attributed to the subjective knee instability relating to reflex quadriceps inhibition secondary to acute anterior knee pain associated with PFJ pathology ${ }^{13,14)}$. This highlights our rationale for selecting the 4 questions forming the PFJ subscore.

Many surgeons use the OKS as a tool for measuring symptom severity of knee arthritis pre-operatively as well as outcome measure in those patients after joint arthroplasty. The OKS is a readily available tool and well-established across practice in the United Kingdom. Selecting questions from this tool, therefore, would allow us to address our question in a non-invasive and efficient approach. Although patients were aware of the study taking place, none had any concerns or objections due to its nature. We feel this is a readily reproducible way of addressing this question in the future across multiple centres.

The average follow-up period for this study was just short of 12 months. Analysis into the OKS has indicated that 12 months of follow-up is sufficient to discern a minimally clinically important difference of 5 points in patients undergoing knee arthroplasty ${ }^{15}$, supporting the overall rigour of our study.

Our findings suggested minor trends correlating the 4 individual questions extracted as well as the combined PFJ subscore to PFJ pathology. From this study, however, our theory that the subscore derived from the OKS could be useful for identifying PFJ pathology and predicting its response to surgery could not be statistically proven from the data we collected. Several reasons 
could be attributed to this outcome. A fundamental limitation of our study lied with the small number of patients recruited for involvement. A retrospective power analysis was conducted using the significance level generated comparing the 4 question PFJ subscore difference between PFA and TFJ arthroplasty. The conventional type 1 error (alpha) significance value of 0.05 was utilized. This generated a power of 0.43 , raising a significant risk of type 2 error in our study. Furthermore, recruitment into our study took place consecutively; however, due to the small number of patients suitable for inclusion across the large study period, randomization was not practically possible raising the risk of selection bias.

We acknowledge that the 4 questions we selected from the OKS are indicative of PFJ pathology only and not confirmative of this pathology. In our study strategy, we attempted to minimize the number of confounding variables through our exclusion criteria including multiple compartment involvement, combined pathology involvement (e.g. associated meniscal or ACL injuries), and patients with clinical patella instability.

We would welcome further studies evaluating the predictive value of radiography, magnetic resonance imaging and arthroscopy in diagnosing isolated compartment involvement and ultimately determining the response to unicompartmental arthroplasty. Furthermore, we would suggest that larger multicenter studies addressing the question in the current article should be performed in the future in order to further assess the importance of symptomatology in the diagnostic work-up of patients being considered for UKA.

\section{Conclusions}

Our study attempted to quantify the relevance of PFJ symptoms in relation to unicompartmental arthroplasty. We were neither able to prove that these symptoms were predictive of PFJ pathology in the preoperative setting, nor were they useful in interpreting which symptoms would likely improve following PFA. The results of our study would support an investigative strategy for patients being considered for UKA with emphasis on preoperative radiographic evaluation to determine knee compartment involvement, followed by either magnetic resonance imaging or arthroscopic evaluation in order to confirm the degree of articular damage, alleviating the degree of importance placed on the preoperative symptomatology.

\section{Conflict of Interest}

No potential conflict of interest relevant to this article was reported.

\section{References}

1. Davies AP, Vince AS, Shepstone L, Donell ST, Glasgow MM. The radiologic prevalence of patellofemoral osteoarthritis. Clin Orthop Relat Res. 2002;(402):206-12.

2. Rand JA. Role of arthroscopy in osteoarthritis of the knee. Arthroscopy. 1991;7:358-63.

3. Insall JN, Joseph DM, Msika C. High tibial osteotomy for varus gonarthrosis: a long-term follow-up study. J Bone Joint Surg Am. 1984;66:1040-8.

4. Matthews LS, Goldstein SA, Malvitz TA, Katz BP, Kaufer H. Proximal tibial osteotomy. Factors that influence the duration of satisfactory function. Clin Orthop Relat Res. 1988;(229):193-200.

5. Ackroyd CE, Newman JH, Evans R, Eldridge JD, Joslin CC. The Avon patellofemoral arthroplasty: five-year survivorship and functional results. J Bone Joint Surg Br. 2007;89:310-5.

6. Rand JA, Ilstrup DM. Survivorship analysis of total knee arthroplasty: cumulative rates of survival of 9200 total knee arthroplasties. J Bone Joint Surg Am. 1991;73:397-409.

7. Newman J, Pydisetty RV, Ackroyd C. Unicompartmental or total knee replacement: the 15-year results of a prospective randomised controlled trial. J Bone Joint Surg Br. 2009;91:52-7.

8. Goudakos IG, Konig C, Schöttle PB, Taylor WR, Singh NB, Roberts I, Streitparth F, Duda GN, Heller MO. Stair climbing results in more challenging patellofemoral contact mechanics and kinematics than walking at early knee flexion under physiological-like quadriceps loading. J Biomech. 2009;42:2590-6.

9. Dawson J, Fitzpatrick R, Murray D, Carr A. Questionnaire on the perceptions of patients about total knee replacement. J Bone Joint Surg Br. 1998;80:63-9.

10. National Joint Resistry. National Joint Registry for England and Wales 7th annual report 2010 [Internet]. Hemel Hempstead: National Joint Resistry; 2010 [cited 2015 Jan 10]. Available from: http://www.njrcentre.org.uk/njrcentre/ portals/0/njr\%207th\%20annual\%20report\%202010.pdf.

11. Davies AP. Predictability of outcome after unicompartmental or total knee replacement. Orthop Proc. 2012; 94(SUPP XXIX):88. 
12. Borus $\mathrm{T}$, Thornhill $\mathrm{T}$. Unicompartmental knee arthroplasty. J Am Acad Orthop Surg. 2008;16:9-18.

13. Thomee R, Renstrom P, Karlsson J, Grimby G. Patellofemoral pain syndrome in young women. I. A clinical analysis of alignment, pain parameters, common symptoms and functional activity level. Scand J Med Sci Sports. 1995;5:237-44.
14. Blazina ME, Fox JM, Del Pizzo W, Broukhim B, Ivey FM. Patellofemoral replacement. Clin Orthop Relat Res. 1979;(144):98-102.

15. Murray DW, Fitzpatrick R, Rogers K, Pandit H, Beard DJ, Carr AJ, Dawson J. The use of the Oxford hip and knee scores. J Bone Joint Surg Br. 2007;89:1010-4. 